\title{
Cbl promotes clustering of endocytic adaptor proteins
}

Daniela Jozic $^{1,5}$, Nayra Cárdenes ${ }^{2,5}$, Yonathan Lissanu Deribe ${ }^{3,5}$, Gabriel Moncalián ${ }^{2}$,

Daniela Hoeller ${ }^{3}$, Yvonne Groemping ${ }^{4}$, Ivan Dikic ${ }^{3}$, Katrin Rittinger ${ }^{1}$ and Jerónimo Bravo $^{2}$

${ }^{1}$ Division of Protein Structure, National Institute for Medical Research, The Ridgeway, London NW7 $1 \mathrm{AA}, \mathrm{UK}$

${ }^{2}$ Signal transduction group, Structural Biology and Biocomputing Programme, Centro Nacional de Investigaciones Oncológicas (CNIO). Melchor Fernández Almagro 3, E-28029 Madrid, Spain.

${ }^{3}$ Institute of Biochemistry II, Goethe University Medical School, Theodor-Stern-Kai 7, 60590 Frankfurt a/M, Germany

${ }^{4}$ Abteilung Biomolekulare Mechanismen, Max-Planck-Institut fuer Medizinische Forschung, Jahnstr. 29, 69120 Heidelberg, Germany

${ }^{5}$ These authors contributed equally to this work

Correspondence should be addressed to K.R. (katrin.rittinger@nimr.mrc.ac.uk), J.B. (jbravo@cnio.es) or I.D. (ivan.dikic@biochem2.de).

Keywords: adaptor protein, CIN85, PIX/Cool, Cbl, protein-protein interaction, Xray crystallography, endocytosis, EGFR downregulation, $\mathrm{SH3}$ domain recognition 
The ubiquitin ligases c-Cbl and Cbl-b play a crucial role in receptor downregulation by mediating multiple monoubiquitination of the receptor and promoting their sorting for lysosomal degradation. Their function is modulated through interactions with regulatory proteins including CIN85 and PIX, which recognize a proline-arginine motif in $\mathrm{Cbl}$ and thus promote or inhibit receptor endocytosis. We report the structures of SH3 domains of CIN85 and $\beta$-PIX in complex with a proline-arginine peptide from Cbl-b. Both structures reveal a heterotrimeric complex containing two SH3 domains held together by a single peptide. Trimerization also occurs in solution and is facilitated by the pseudosymmetrical peptide sequence. Moreover, ternary complexes of CIN85 and Cbl are formed in vivo and are important for the ability of Cbl to promote EGFR downregulation. These results provide molecular explanations for a novel mechanism by which $\mathrm{Cbl}$ controls receptor downregulation. 


\section{INTRODUCTION}

Ligand binding to receptor tyrosine kinases (RTKs) causes receptor activation and initiates intracellular signalling cascades, which ultimately control biological responses such as cell proliferation, cellular adhesion or apoptosis ${ }^{1}$. In order to regulate the lifetime of this process, activated RTKs also trigger negative feedback loops that inhibit receptor activation or promote receptor endocytosis and lysosomal degradation ${ }^{2}$. The latter process is mediated by the formation of large protein complexes capable of removing activated receptors from the plasma membrane via clathrin-dependent and independent pathways ${ }^{3-6}$. In this process, $\mathrm{c}-\mathrm{Cbl}$ and $\mathrm{Cbl}-\mathrm{b}$ proteins play a critical role by acting as ubiquitin ligases as well as endocytic adaptor molecules. Cbl-mediated multiple monoubiquitination of RTKs sorts the receptor containing vesicle to a degradation route and thereby leads to termination of the RTK signal ${ }^{7-9}$. Upon cell stimulation, $\mathrm{Cbl}$ proteins get translocated to activated RTKs and act as multifunctional adaptor proteins, capable of recruiting additional endocytic regulatory proteins such as CIN85-endophilin complexes, which facilitate receptor endocytosis.

A major function of CIN85 arises from its ability to bind to Cbl proteins as well as other effectors involved in regulation of receptor endocytosis ${ }^{10-12}$. Complex formation between CIN85 and Cbl occurs via the selective binding of SH3 domains of CIN85 to an atypical proline-arginine motif (PxxxPR) present in the $\mathrm{C}$-terminal region of $\mathrm{c}-\mathrm{Cbl}$ and Cbl-b, and other CIN85 effectors ${ }^{13,14}$. Since CIN85 has three SH3 domains (SH3A, B and C; Fig. 1) that all recognize the same motif, it was proposed that CIN85 acts as a platform for clustering of $\mathrm{Cbl}$ molecules thereby contributing to efficient degradation of activated epidermal growth factor receptors (EGFRs) ${ }^{13}$. The closest homologue of CIN85-SH3A (from now on referred to as CIN85A) outside of the CIN85/CMS protein 
family is $\beta$-PIX, which has $52 \%$ sequence identity (Fig. 1). Interestingly, $\beta$-PIX has previously been shown to bind to a region of p21-activated kinase (Pak) that also contains an atypical proline-arginine motif ${ }^{15}$. Functionally, PIX proteins are believed to act as guanine nucleotide exchange factors (GEFs) for the small GTPases Rac and $\mathrm{Cdc} 42^{15,16}$, which regulate a multitude of cellular processes including cell polarity and motility, gene transcription and phagocytosis ${ }^{17}$. Recently, $\beta$-PIX/Cool-1 has been described as another Cbl-interacting protein that modulates EGFR downregulation ${ }^{18}$. It was shown that activated Cdc42-PIX complexes promote sequestration of $\mathrm{Cbl}$ from the EGFR and thereby block EGFR endocytosis ${ }^{19}$. The underlying mechanism has yet to be unravelled, but our recent findings imply that $\beta$-PIX binds to the proline-arginine motif in $\mathrm{Cbl}$, and thus may compete with CIN85 for binding to $\mathrm{Cbl}$ (data not shown).

SH3 domains are classic examples of protein interaction modules. They generally bind to proline-rich sequences containing a core PxxP motif, although other sequences may also be recognized ${ }^{20-23}$. Many ligands bind their respective SH3 domains in a polyproline II helix (PPII) conformation ${ }^{24}$. Due to the twofold rotational pseudosymmetry of this conformation, peptides can bind a given $\mathrm{SH} 3$ domain in either one of two opposite directions governed by the location of a positively charged residue, usually Arg, which precedes or follows the core PxxP motif ${ }^{25,26}$. SH3 domains associate with their ligands with a 1:1 stoichiometry and proteins containing multiple SH3 domains can either serve as adaptor proteins to co-localize multiple proteins or can bind multivalent ligands and thereby increase the affinity and specificity of a given interaction. An exception is the so-called 'SuperSH3' domain, first noted in the NADPH oxidase subunit $\mathrm{p} 47^{\text {phox }}$, where two tandem SH3 domains co-operate to bind a single $\operatorname{ligand}^{27}$. This configuration appears to constitute a novel protein interaction module that provides greater flexibility for the recognition of multiple, diverse ligands. 
To understand the functional role of complex formation between $\mathrm{Cbl}$ and CIN85 or $\beta$-PIX, we investigated these interactions in vitro and in vivo. Crystal structures of complexes between a Cbl-b peptide and SH3 domains of either human CIN85 or rat $\beta$ PIX reveal that, somewhat surprisingly, the Cbl-peptide can interact simultanously with two molecules of either SH3 domain. We demonstrate, by isothermal titration calorimetry (ITC) that heterotrimerization also occurs in solution and is dependent on the presence of an N-terminal arginine residue in the Cbl-b peptide. Structural analysis of these complexes shows that they are distinct from those formed by 'SuperSH3' domains and explains why other targets of CIN85 or PIX do not support heterotrimerization. Finally we provide evidence that Cbl-induced binding to two CIN85 molecules also occurs in vivo, indicating a novel role of $\mathrm{Cbl}$ in mediating formation of larger protein complexes involved in regulation of receptor endocytosis. 


\section{RESULTS}

\section{Overall structures of trimeric SH3-peptide complexes}

Structures of complexes of CIN85A and the $\beta$-PIX SH3 domain each in complex with proline-arginine motif-containing peptides derived from Cbl-b were solved by molecular replacement and refined at a resolution of 2.0 and $1.85 \AA$, respectively (Table 2). Each protein-peptide assembly crystallizes as a ternary complex that is composed of two SH3 domains and a single Cbl-b peptide (Fig. 2a and 2b). Both SH3 domains adopt the typical SH3 domain fold, a five stranded $\beta$-sandwich, and overlap with one another with rms deviations between $\mathrm{C} \alpha$ atoms of 0.8-1.2 $\AA$. In either of the heterotrimeric complexes, two SH3 domains contact the Cbl-b peptide, that itself adopts a classical PPII helical conformation, through their conserved ligand-binding surfaces. However, one $\mathrm{SH} 3$ engages the peptide in a class I interaction (defined as $\mathrm{SH} 3_{\mathrm{A}}$ ) while the other binds on the opposite side of the peptide ligand in a class II orientation (defined as $\left.\mathrm{SH} 3_{\mathrm{B}}\right)$. The peptide is thus sandwiched by the two $\mathrm{SH} 3$ domains in the ternary complex and there are no direct SH3-SH3 contacts in the CIN85A/Cbl-b structure. In the PIX/Cbl-b complex direct contacts bury $73 \AA^{2}$ in and are made through van der Waals contacts between PIX Asn58 from each domain.

\section{Peptide binding and heterotrimerization}

Complex formation between the Cbl-b peptide and either PIX SH3 domain buries $771 \AA^{2}\left(\mathrm{SH}_{\mathrm{A}}\right)$ and $776 \AA^{2}\left(\mathrm{SH} 3_{\mathrm{B}}\right)$. These values are comparable to those of the equivalent CIN85A/Cbl-b complex (928 and $715 \AA^{2}$, respectively) and generally to those of other SH3 domain-peptide structures. The SH3-peptide interactions are summarized in Figure 2c (for PIX) and 2d (for CIN85A). A large number of hydrogen 
bonds are formed by backbone carbonyl oxygens of the peptide to side chains of either SH3 domain. In the PIX/Cbl-b complex these interactions include Cbl-b Pro905 with Trp43 $_{\text {A }}$, Pro906 with Tyr59 B, Lys907 with Asn58 of each subunit, Pro908 with Tyr59 and Arg909 with Trp43 B. Specific interactions via side chains are only made by Arg904 and Arg911 from Cbl-b at the extreme N-and C-termini of the peptide with Asp23 and Glu24 of each SH3 domain, respectively. Pro906 and Pro910 point into hydrophobic pockets made by Phe15 and Tyr59 of either SH3 domain (Pro906 towards $\mathrm{SH}_{\mathrm{B}}$ and Pro910 toward $\mathrm{SH}_{\mathrm{A}}$ ), while Pro908 is surrounded by Tyr59, Phe17, Pro56 and Trp43 from $\mathrm{SH} 3_{\mathrm{B}}$.

Equivalent interactions are present in the CIN85A/Cbl-b complex. Specifically, Cbl-b Pro905 interacts with Trp36 , Lys907 with Asn51 of each SH3 subunit and Arg909 with Trp36 $6_{\mathrm{B}}$. The side chain of Arg904 interacts with Asp16 ${ }_{\mathrm{A}}$ and Glu17 $\mathrm{A}$ while Arg911 makes contacts with Asp16 and Glu17 from molecule B. Cbl-b prolines 906 and 910 point into hydrophobic pockets made by Phe8 and $\mathrm{Phe} 52$ of $\mathrm{SH} 3_{\mathrm{B}}$ and $\mathrm{SH} 3_{\mathrm{A}}$ respectively and Pro908 is surrounded by Phe52, Tyr10, Pro49 and Trp36 from SH3 $3_{B}$.

Lys907 from the Cbl-peptide plays a central role in both complexes. It is the only residue that makes simultaneous contacts with both $\mathrm{SH} 3$ domains through hydrogen bonds from its backbone carbonyl to the side chain amides of each, Asn58 in PIX/Cbl-b or Asn51 in CIN85A/Cbl-b. The contacts made between the Cbl-peptide and either SH3 domain are pseudo-symmetrical and the backbone carbonyl of Lys907 constitutes the pseudo dyad axis of these interactions. Accordingly, equivalent interactions are established by Arg904 and Arg911 as well as Pro905 and Arg909 from Cbl-b (Fig. 2). The pseudo-symmetry of interactions is slightly disrupted around Pro906, which makes an analogous hydrogen bond as Pro908 (to Tyr59 from PIX), while at the same time being in a similar hydrophobic environment as Pro910. The main difference between 
the PIX/Cbl-b and CIN85A/Cbl-b complexes is that Tyr59 in PIX is a Phe in CIN85A and hence there are no hydrogen bonds to the backbone carbonyls of Pro906 and Pro908.

\section{Heterotrimeric complex formation in solution}

The heterotrimeric CIN85A/Cbl-b and PIX/Cbl-b complexes described here are, so far as we are aware, the first examples of peptide-induced dimerization of SH3 domains. In order to ensure that this is a general process that occurs in solution and is not solely an artifact of crystallization we analyzed the SH3 domain-peptide interactions by ITC. Binding of the Cbl-b peptide to PIX-SH3 resulted in a curve that could be fit to a single site binding model with an apparent dissociation constant of $14 \mu \mathrm{M}$ and a stoichiometry of 1:0.57 (Table 1), indicating that approximately only half the amount of peptide is required to form a complex with PIX. The same observation was made upon titration of CIN85A with the Cbl-peptide: binding occurred with a stoichiometry of 1:0.57 and an apparent $\mathrm{K}_{\mathrm{d}}$ of $2.5 \mu \mathrm{M}$ (Table 1 and Fig. 3). In contrast, titration of PIXSH3 with a peptide derived from Pak $1^{15}$, resulted in complex formation with a stoichiometry of 1:0.95 and a $\mathrm{K}_{\mathrm{d}}$ of $3.2 \mu \mathrm{M}$ (Table 1). Pak2 has been identified as a binding partner of $\mathrm{CIN} 85^{14}$. However the affinity of the Pak1-derived peptide, which is highly homologous to Pak2, for CIN85A was rather weak ( $\mathrm{K}_{d}$ of $\left.68 \mu \mathrm{M}\right)$, supporting the notion that CIN85B may be the preferred ligand for Pak ${ }^{14}$. Nevertheless, given the high protein and peptide concentrations used in this titration, the data could be fit reliably and showed a 1:1 stoichiometry (Table 1, data not shown).

Previous studies have indicated that Arg911 in Cbl-b (PxxxPR) is important for the interaction with $\mathrm{CIN} 85^{13,14}$, an observation which can now be rationalized by the multiple hydrogen bonds formed between its guanidinium group and Asp16/Glu17 from 


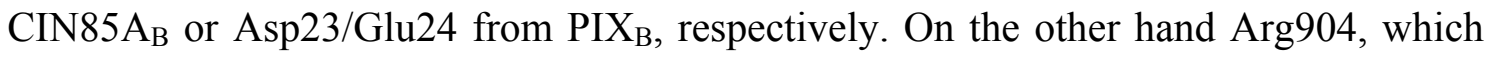
makes equivalent contacts with $\mathrm{CIN} 5 \mathrm{~A}_{\mathrm{A}}$ and $\mathrm{PIX}_{\mathrm{A}}$ has not been previously implicated in complex formation. Our structures suggest that this residue may be crucial to allow peptide induced dimerization, an idea that is supported by the observation that this arginine is absent in the Pak-peptide, which binds either SH3 domain with a 1:1 stoichiometry. To test this hypothesis we measured binding of a mutant Arg904Ala Cblpeptide to CIN85A and PIX. Titration of CIN85A with this peptide clearly showed that stoichiometric amounts are required to saturate the SH3 domain with an affinity of 4.4 $\mu \mathrm{M}$, further supporting our model (Fig. 3 and Table 1). In contrast, mutation of Arg904 greatly weakened the interaction with PIX to $140 \mu \mathrm{M}$, thereby making the determination of the stoichiometry of the interaction somewhat unreliable. Nevertheless, considerably more than half an equivalent of mutant peptide is required to approach saturation suggesting that this interaction also occurs with a 1:1 stoichiometry.

The stoichiometry of the CIN85A/Cbl-b complex described in this manuscript differs from an earlier study by Kowanetz and colleagues, which reported a 1:1

complex $^{13}$. At present we are not certain about the reasons for this discrepancy but believe that it is most likely due to the experimental conditions and the size of the constructs used, which differ extensively between the two studies.

\section{In vivo interactions between $\mathrm{c}-\mathrm{Cbl} / \mathrm{Cbl}-\mathrm{b}$ and $\mathrm{CIN85}$}

Previous findings have indicated that the C-terminal leucine zipper (LZ) of c-Cbl mediates protein dimerization and thereby formation of larger complexes needed for efficient EGF receptor downregulation ${ }^{28}$. The data presented here suggest that $\mathrm{Cbl}$ proteins may additionally contribute to the formation of such complexes by simultaneously interacting with two CIN85-SH3 domains. We first tested whether c-Cb1 
that also contains the pseudo-symmetrical proline-arginine motif can promote ternary complex formation with CIN85 in vivo by co-transfection of full-length CIN85 and FLAG-CIN85-3SH3 with different c-Cbl mutants in HEK293T cells. FLAG-CIN853SH3 (a construct containing the three SH3 domains of CIN85) is impaired in its ability to oligomerize due to the lack of the coiled-coil motif and hence cannot form heterocomplexes with wild type $\mathrm{CIN} 85^{13}$. This makes it particularly useful to monitor interactions between $\mathrm{SH} 3$ domains of CIN85 in the presence or absence of a potential linker such as c-Cbl. Accordingly, co-precipitation between CIN85 and FLAG-CIN853SH3 was detected at very low levels in cells, likely due to the presence of endogenous c-Cbl (Fig. 4a). On the other hand, co-precipitation was increased upon co-expression with c-Cbl deleted for the LZ domain (c-Cbl-849), which retained its ability to bind to FLAG-CIN85-3SH3 (Fig. 4a, upper panel). This mutant was used to ensure that coprecipitation was a consequence of binding of two independent CIN85 molecules to a single c-Cbl molecule rather than due to the interaction with homodimeric c-Cbl ${ }^{28}$. More importantly, a c-Cbl mutant (c-Cbl-827), which is deleted for the $\mathrm{LZ}$ domain and the proline-arginine binding site, could not interact with SH3 domains of CIN85 and thus did not promote co-precipitation between FLAG-CIN85-3SH3 and wild type CIN85 (Fig. 4a, lower panel). Since the crystal structures and ITC studies presented here implicate that Arg904 in the Cbl-b peptide is crucial for allowing its heterotrimerization with $\mathrm{CIN} 85 \mathrm{SH} 3 \mathrm{~A}$, we analyzed the role of this residue on the in vivo interaction between CIN85 and Cbl-b (Fig. 4b). As shown in Fig. 4b, Cbl-b deleted for the LZ/UBA domain (Cbl-b-927), which is unable to homodimerize, interacted potently with SH3 domains of CIN85, whereas Cbl-b-927 R904A was reduced, but not blocked, in its ability to co-precipitate with FLAG-CIN85-3SH3. Furthermore, the Cbl-b-927 R904A mutant was impaired in its ability to mediate 
formation of a complex between full-length CIN85 and FLAG-CIN85-3SH3 (Fig. 4b). We noticed that c-Cbl was consistently more potent, compared to Cbl-b, in inducing trimerization with CIN85 (Fig. 4a and 4b). However, the pseudo symmetrical prolinearginine motif is almost identical in both $\mathrm{Cbl}$ isoforms indicating that the reason for this effect must be due to differences in other regions of the two proteins. Due to its stronger binding, we used c-Cbl to quantify the relative contributions of the two conserved arginines, Arg822 and Arg829, for interaction with and trimerization of CIN85. As shown in Fig. 4c mutation of either Arg822 or Arg829 considerably reduced their ability to promote simultaneous binding to full-length CIN85 and FLAG-CIN85-3SH3 supporting the conclusion that an intact pseudo-symmetrical proline-arginine motif is required to allow $\mathrm{Cbl}$ proteins to promote heterotrimeric complex formation in vivo. However, heterotrimerization was not completely abolished suggesting that in cells either additional interacting partners of $\mathrm{c}-\mathrm{Cbl}$ and CIN85 may indirectly contribute to heterologous complex formation or that regions outside the core binding motif further stabilize this complex.

To determine the functional role of the individual arginines in full-length c-Cbl and Cbl-b for binding to CIN85 and receptor downregulation, we mutated either Arg822 or Arg829 in c-Cbl, or Arg904 or Arg911 in Cbl-b separately or both of them simultaneously. The amount of CIN85 co-precipitated with c-Cbl and Cbl-b proteins was quantified and showed that mutation of Arg829 and Arg911 reduced the interaction with CIN85 by approximately 80 and $60 \%$ respectively, whereas mutation of Arg 822 or Arg904 reduced their binding by approximately $25 \%$. Mutation of both sites in either cCbl or Cbl-b largely abolished their co-precipitation with CIN85 (Fig. 4d). Lastly, we tested the functional importance of these arginines by measuring the downregulation rate of EGF receptors in cells transfected with different $\mathrm{Cbl}$ mutants. Expression of 
dimerization-deficient c-Cbl-849 or Cbl-b-927 in HEK293 cells enhances EGF-induced degradation of EGF receptors (Fig. 4e and 4f). This effect was substantially reduced if either of the two arginine residues were mutated, indicating that both play important roles in pathways controlling EGF receptor downregulation. These findings together with our data on heterotrimerization of CIN85 by c-Cbl and Cbl-b (Fig. 4a-c) point to an important and previously unaccounted role of Cbl-b Arg904 and c-Cbl Arg822 in Cbl-mediated clustering of CIN85 and controlling endocytic trafficking of EGF receptors. 


\section{DISCUSSION}

It has become evident that the interaction of $\mathrm{c}-\mathrm{Cbl} / \mathrm{Cbl}-\mathrm{b}$ with adaptor proteins such as CIN85 and PIX is an important mechanism to modulate and fine-tune RTK endocytosis and degradation ${ }^{2}$. The same proline-arginine motif in the C-terminal portion of $\mathrm{c}-\mathrm{Cbl} / \mathrm{Cbl}-\mathrm{b}$ is recognized by $\mathrm{SH} 3$ domains present in both proteins, suggesting that they may act in a competitive fashion with respect to $\mathrm{Cbl}$. We report here the crystal structures of complexes between a Cbl-b proline-arginine peptide and the SH3 domains of $\beta$-PIX and CIN85 (SH3A), respectively. Unexpectedly, the Cbl-peptide induces formation of a heterotrimeric complex consisting of two SH3 domains and one peptide molecule in both structures. Interactions made by the Cbl-peptide with $\beta$-PIX and CIN85A are very similar and the peptide binds simultaneously in class I and II orientations. Key to this unusual arrangement is the pseudo-symmetry of the peptide sequence that allows two arginine residues, Arg904 and Arg911, to make equivalent hydrogen bonds with acidic residues in each SH3 domain. This pseudo-symmetry is also preserved in c-Cbl due to the presence of Arg822 and Arg829. The previously shown requirement of Arg911 for complex formation can now be rationalized by the extensive interactions between this residue and the $\mathrm{SH} 3$ domain: the guanidinium side chain is involved in a network of hydrogen bonds with acidic residues in the RT-loop while its aliphatic portion stacks against Trp43 in PIX and Trp36 in CIN85. Arg904 on the other hand has not been previously recognized as important for complex formation with CIN85. Its presence appears to define the multimerization state of the SH3/peptide complexes and hence provides a rational explanation why other ligands that also contain the PxxxPR motif, but lack the N-terminal arginine such as Pak, bind with a 1:1 stoichiometry. Furthermore, it suggests that other targets of CIN85 that lack the Nterminal arginine such as BLNK or CD2 should bind in a 1:1 fashion. Interestingly, 
mutation of this arginine does not appreciably change the apparent affinity of the peptide for CIN85A, but changes the relative enthalpic and entropic contributions to $\Delta \mathrm{G}$. In contrast, binding to PIX-SH3 is weakened 10-fold upon mutation of Arg904 indicating that despite of the similarity between the two structures there must be differences in the mechanism of complex formation. Intriguingly, in vivo, neither mutation of Arg822 nor Arg829 (the equivalent residues in c-Cbl) completely abolishes heterotrimerization, which may indicate that in the context of the full-length proteins there might be residues outside of the core motif that contribute to the stabilization of a heterotrimeric complex or alternatively that additional interacting proteins may indirectly contribute to their co-precipitation by forming multimeric complexes.

\section{Comparison with SuperSH3 domains}

The PIX/Cbl and CIN85/Cbl structures are at present the only examples of ternary SH3 domain/peptide complexes, in which the peptide induces dimerization and potentially oligomerization of its targets. The only other examples of SH3/ligand complexes, in which the ligand is shared between two SH3 domains is that of $\mathrm{p} 47^{\text {phox }}$ in the autoinhibited and the p22 $2^{\text {phox }}$-bound state, a structural arrangement that has been termed the 'SuperSH3' domain ${ }^{27}$. However, in contrast to the structures described here, the SH3 domains of $\mathrm{p} 47^{\text {phox }}$ are covalently linked and this link is required to allow the two SH3 domains to simultaneously bind the same peptide. While the individual SH3 domains of the $\mathrm{p} 47^{\mathrm{phox}}$ and PIX or CIN85 structures overlap well with one another, an overlap of the trimeric PIX/Cbl structure with that of the dimeric $\mathrm{p} 47-\mathrm{p} 22^{\text {phox }}$ complex shows that the second SH3 domain in each structure occupies a different position (Fig. 5). In the PIX/Cbl-b and CIN85A/Cbl-b complexes the two SH3 domains come closest at PIX Asn58 and CIN85 Asn51 situated in a 310 helix in the C-terminal portion of the 
proteins (Fig. 1 and 2). In p47 $7^{\text {phox }}$ it is the n-Src loops, which come closest, bury $579 \AA^{2}$ of solvent accessible surface (through direct domain interactions) and contribute to the stability of the SuperSH3 domain conformation through hydrogen bonds across the interface. A "GWW" motif present in each n-Src loop acts as a signature motif of SuperSH3 domains ${ }^{27}$. The glycine residue is required for steric complementarity while the second tryptophan residue makes important interactions across the interface. Interestingly, PIX as well as all three SH3 domains of CIN85 contain this motif (Figure1). Nevertheless, their relative orientation in the heterotrimeric structures described here is different from that observed in $\mathrm{p} 47^{\text {phox }}$ and residues of the $\mathrm{n}$-Src loop do not contribute to trimerization. This suggests that the new structures presented here are not additional examples of SuperSH3 domains but rather constitute a novel mechanism by which $\mathrm{SH} 3$ domains can contribute to the formation of multiprotein complexes.

\section{Cbl mediated protein multimerization in endocytosis}

Efficient receptor endocytosis requires the formation of large protein complexes surrounding activated receptors ${ }^{4,29}$. In many instances, specialized endocytic scaffold proteins engage multiple effectors and couple trafficking cargo with the clathrin coat, actin cytoskeleton and endocytic vesicles. Among them are $\mathrm{Cbl}$ proteins that interact with a CIN85/endophilin complex and control receptor internalization ${ }^{4}$. Previously it was shown that CIN85 contributes to receptor internalization by clustering Cbl proteins via its three $\mathrm{SH} 3$ domains ${ }^{13}$. However, findings presented here reveal yet another mechanism by which $\mathrm{Cbl}$ proteins can contribute to the formation of signal competent complexes implicated in receptor endocytosis. Biochemical studies in cells clearly indicate that the proline-arginine motif in $\mathrm{Cbl}$ can simultaneously accommodate two 
SH3 domains from separate CIN85 molecules (Fig. 4) and could thereby promote the formation of higher order Cbl-CIN85 complexes. At present we cannot exclude that adjacent SH3 domains in CIN85 may act in a similar manner and co-operate to bind a single ligand. Further studies are required to understand such a potential interplay between $\mathrm{SH} 3 \mathrm{~A}, \mathrm{~B}$ and $\mathrm{C}$ in the context of the full-length protein. Nevertheless, consistent with our findings, high molecular complexes containing Cbl and CIN85 have been found in cellular lysates ${ }^{30}$. Furthermore, the identification of Cbl-b Arg904 as an additional binding surface for SH3 domains allows to explain how Cbl-mediated multimerization of CIN85 proteins occurs in vivo. The physiological importance of these $\mathrm{Cbl}$ functions is likely to be relevant in the assembly of multi-protein complexes during receptor downregulation ${ }^{13,28,31}$. It is also important to notice that the $\beta$-PIX-SH3 domain binds in the same manner to Cbl proteins as CIN85. Thus, the competition of $\beta$ PIX and CIN85 for binding to the same binding surface of $\mathrm{Cbl}$ may represent the basis for dissociation of $\mathrm{Cbl}$ away from the activated EGF receptor upon activation of Cdc42PIX complexes ${ }^{19}$.

Taken together, we have identified critical residues in the proline-arginine motif of $\mathrm{c}-\mathrm{Cbl} / \mathrm{Cbl}-\mathrm{b}$ involved in ternary complex formation with the adaptor proteins CIN85 and $\beta$-PIX and the assembly of the endocytic machinery that governs downregulation of activated receptor tyrosine kinases. Future research should now be directed towards the visualization and dynamics of the formation of Cbl-linked protein complexes in cells and their live imaging during receptor trafficking in the endosome. 


\section{METHODS}

Protein overexpression and purification. CIN85A: The N-terminal SH3 domain of human CIN85 (residues 1-58) was cloned into pET29b and overexpressed in E. Coli strain Rosetta(DE3)pLysS at $30^{\circ} \mathrm{C}$. Cell lysates were precipitated with $50 \%$ ammonium sulfate $(\mathrm{w} / \mathrm{v})$, the precipitate was discarded by centrifugation at 35,000 g for 15 minutes and more ammonium sulfate was added to the supernantant to $70 \%$ saturation. After centrifugation, the CIN85A-containing supernatant was adjusted to $50 \%$ ammonium sulfate, loaded onto a 5ml HiTrap Phenyl FF (high sub) hydrophobic interaction column (Amersham Biosciences) and eluted with a linear ammonium sulfate gradient (50\% to 0\%). CIN85A containing fractions were pooled, concentrated and loaded onto a HiLoad 16/60 Superdex 75 gel filtration column equilibrated with buffer B (20 mM Tris $\mathrm{pH} 7.5$, $150 \mathrm{mM} \mathrm{NaCl})$.

$\beta$-PIX-SH3: The SH3 domain of rat $\beta$-PIX (residues $5-66$ ) ${ }^{15}$ was cloned into pGEX-4T1 and expressed in E.coli strain BL21 at $30^{\circ} \mathrm{C}$. The protein was purified on glutathione sepharose 4B (Amersham Biosciences) and cleaved on-column with human $\alpha$ Thrombin protease (HTI). Further purification was carried out by gel filtration on Superdex 75 (Amersham Biosciences) in $20 \mathrm{mM}$ Tris $\mathrm{pH} 7.5,50 \mathrm{mM} \mathrm{NaCl}$. The purified domain was concentrated in VivaSpin concentrators (VivaScience) to around 1 $\mathrm{mM}$ and stored at $-70^{\circ} \mathrm{C}$ after shock freezing in liquid nitrogen.

Isothermal titration calorimetry measurements. Complex formation between the SH3 domains of PIX and CIN85 and various peptides was measured by ITC using a MicroCal VP-ITC Microcalorimeter (MicroCal Inc Inc., Northampton, MA) ${ }^{32}$. The peptides used were Cbl-b: SQAPARPPKPRPRRTAY; the C-terminal Tyr has been introduced to allow spectrophotometric determination of the peptide concentration and 
Pak: DATPPPVIAPRPEHTKSVYTR. A NAP 5 column was used to exchange all proteins into ITC buffer $(50 \mathrm{mM}$ Tris or Hepes $\mathrm{pH} 7.5,50 \mathrm{mM} \mathrm{NaCl})$. Experiments were performed at $18^{\circ} \mathrm{C}$. In general the sample cell contained either SH3 domain at concentrations ranging from $20-200 \mu \mathrm{M}$ and peptide solutions at approximately ten times the protein concentration in the cell were injected in $10 \mu 1$ samples from a total of $290 \mu \mathrm{l}$. Heats of dilution, determined by titrating the peptides into buffer alone, were subtracted from the raw titration data before analysis. Data were fitted by least-squares procedures assuming a one site binding model using the evaluation software, Microcal Origin version 7.0 provided by the manufacturer. All measurements were repeated two to four times (apart from those mentioned in the figure legend).

Crystallization, Data Collection, Structure Determination and Refinement. $\beta$-PIXSH3/Cbl-b peptide (same peptide as used for the ITC studies) crystals were obtained by sitting drop vapor diffusion against 0.2 M Sodium Acetate, 30\% PEG 8000, $0.1 \mathrm{M}$ Sodium cacodylate trihydrate, $\mathrm{pH} 6.5$ at $18^{\circ} \mathrm{C}$ with a protein concentration of $118 \mu \mathrm{M}$ and a protein:peptide ratio of $1: 2$ in the drop. For cryoprotection $15 \%$ glycerol was added. The crystals grew in the trigonal space group $\mathrm{P} 3{ }_{2} 21$ with two protein molecules and one peptide/AU. Data were collected at beamline 14.2 at Daresbury laboratories at $100 \mathrm{~K}$ and processed using the HKL program package ${ }^{33}$. The structure was solved by molecular replacement using the program $\mathrm{MOLREP}^{34}$ with the $1 \mathrm{SEM}$ structure $^{26}$ as a search model. An automatic water search/refinement was performed with ARP/REFMAC ${ }^{35,36}$ showing clearly the position of Cbl-b. The model of the complex was built in MAIN ${ }^{37}$ followed by cycles of refinement in REFMAC. The data were refined to $1.85 \AA$ resolution containing amino acids $6-65$ and $3-66$ of $\beta$-PIX and $904-$ 911 of Cbl-b. The stereochemical quality of the protein model was constantly monitored 
during the course of refinement with PROCHECK ${ }^{38}$. The protein model is of excellent stereochemical quality, with all residues lying in the preferred $\Phi / \Psi$ regions.

The Cbl-b peptide (PARPPKPRPRR, Sigma Genosys) was added to CIN85A at a 1:2.5 protein:peptide ratio and $6.96 \mathrm{mM}\left(47.1 \mathrm{mg} \mathrm{ml}^{-1}\right)$ final concentration in $20 \mathrm{mM}$ TrisHCl $\mathrm{pH} 7.5,150 \mathrm{mM} \mathrm{NaCl}$ ). Crystals were obtained by hanging drop vapour diffusion by mixing $0.5 \mu \mathrm{l}$ of protein solution with $0.5 \mu \mathrm{l}$ of reservoir solution $(0.8 \mathrm{M} \mathrm{Na}$ Citrate, 0.1 M Bis-Tris $\mathrm{pH} 7.5,0.2 \mathrm{M} \mathrm{NaCl})$. For cryoprotection $10 \%(\mathrm{v} / \mathrm{v})$ ethylene glycol was added. A $2.0 \AA$ resolution dataset was collected at $100^{\circ} \mathrm{K}$ on a rotating anode. Diffraction data were reduced using the HKL package, DENZO and SCALEPACK ${ }^{33}$. Crystals of CIN85A:Cbl-b belong to space group P65. A twining fraction between 25 and $31 \%$ was detected. Initial phases for CIN85A:Cbl-b were calculated from a single $\mathrm{AMoRe}^{39}$ molecular replacement solution with correlation coefficient and Rfactor 24.1/47.6 \% by using a previously solved isolated CIN85A structure as search model (to be published). The final model was obtained by combining cycles of automatic refinement in $\mathrm{CNS}^{40}$ (considering a twinning fraction of 0.305 for the operator $\mathrm{k}, \mathrm{h},-1$ ) and manual model building using $\mathrm{COOT}^{41}$ or XTALVIEW/XFIT ${ }^{42}$. Residue 58 in CIN85 $\mathrm{A}_{\mathrm{A}}$ is disordered. 82 water molecules have been added to the final model. PROCHECK $^{38}$ indicates that $99 \%$ percent of the residues are in the allowed regions of $\Phi$ and $\Psi$ conformational space.

Biochemical assays and EGF receptor degradation assay. Cell transfection with indicated cDNAs, cell lysis, immunoprecipitation and immunoblotting were performed as previously described ${ }^{13}$. The CIN85 antibody is a rabbit polyclonal antibody directed against the $\mathrm{C}$ terminus of CIN85 and hence does not recognize the FLAG-CIN85-3SH3 construct. Immunoblots were quantified using the NIH ImageJ software ${ }^{43}$. Degradation 
assays were performed as described recently ${ }^{30}$. Point mutations in c-Cbl and Cbl-b were introduced using a modified Stratagene QuikChange mutagenesis protocol and verified by DNA sequencing. 293T cells were transfected with EGFR-GFP and either Cbl-b927, Cbl-b-927 R904A, Cbl-b-927 R911A or empty vector (control) in $10 \mathrm{~cm}$ cell culture dishes. After $24 \mathrm{~h}$ cells were split into 12-well dishes and starved overnight in serum-free medium. The next day cells were left unstimulated or were incubated with EGF (50 ng ml${ }^{-1}$ ) for 60 or $120 \mathrm{~min}$ at $37^{\circ} \mathrm{C}$. Cells were harvested after stimulation and analyzed using the Epics XL flow cytometer (Beckman Coulter). For each sample 10.000 GFP-positive cells were gated and the intensity of green fluorescence determined. Equal expression of the transfected proteins was checked by immunoblotting.

\section{Coordinates}

The atomic coordinates have been deposited in the Protein Data Bank with accession codes 2AK5 ( $\beta$-PIX/Cbl-b) and 2BZ8 (CIN85A/Cbl-b).

\section{ACKNOWLEDGEMENTS}

The $\beta$-PIX-SH3 plasmid was a kind gift of E. Manser (Singapore). We are grateful to S. Howell and L. Haire (NIMR) for mass spectrometry, P. Fletcher (NIMR) and W. Mawby (University of Bristol) for peptide synthesis, J. Nicholson and R. Kehoe at Daresbury Laboratory for beamline assistance, A. Albert for help in data collection and M. Ortiz for help with CIN85A structure refinement. Research on CIN85 was supported by grant SAF2003-03860 of the Ministerio de Ciencia y Tecnología, Spain and N.C. received a fellowship from the Ramón Areces Foundation. D.J. and K.R. are funded by 
the Medical Research Council, UK. Work by Y.L.D, D.H. and I.D. are supported by grants from Deutsche Forschungsgemeinschaft, Germany.

\section{Competing interests statement}

The authors declare that they have no competing financial interests. 


\section{FIGURE LEGENDS}

Figure 1. Domain structure of $\beta$-PIX and CIN85 and sequence alignment of SH3 domains. Schematic showing the overall domain structure of $\beta$-PIX and CIN85 (DH: Dbl homology domain, PH: pleckstrin homology domain, P-rich: proline rich, GBD: Git binding domain). CIN85 and $\beta$-PIX are both believed to multimerize via their respective coiled-coil (CC) domains (yellow). The sequence alignment was carried out between the SH3 domains of CIN85A, $\beta$-PIX, p47 $7^{\text {phox }}$ and Sem-5. Residues that make contacts with the Cbl-peptide are highlighted in red and residues that are completely conserved in blue. The position of the GWW motif is indicated by asterisks.

Figure 2. Overall structure of the $\beta$-PIX/Cbl-b and $\mathrm{CIN} 85 \mathrm{~A} / \mathrm{Cbl}-\mathrm{b}$ heterotrimeric complexes and details of the protein-peptide contacts. Ribbons representation of the biologically relevant heterotrimeric complex of $\beta$-PIX and Cbl-b (a) colored in green and the trimeric complex of CIN85A and Cbl-b (b) colored in blue. The electron density map around the peptide is contoured at $2.5 \sigma$. The secondary structural elements and position of the RT, n-Src and distal loops are indicated. Both structures are shown in the same orientation to highlight their similarity. The peptide in the CIN85A complex shows additional density for residues Ala903, Pro902 and Arg912, which are disordered in the PIX complex. A schematic representation of the protein-peptide contacts between the Cbl-peptide and $\beta$-PIX and CIN85A are shown in (c) and (d), respectively. The peptide is colored in green (PIX/Cbl-b) and blue (CIN85/Cbl-b) and residues from $\beta$ PIX and CIN85A in black. Hydrogen bonds are indicated by black dashed lines and hydrophobic contacts by black rays. The relevant $\mathrm{SH} 3$ domains are indicated in brackets. 
Figure 3. Isothermal titration calorimetry measurements of complex formation between (a) CIN85A $(30 \mu \mathrm{M})$ and the wildtype Cbl-peptide $(333 \mu \mathrm{M})$ and (b) CIN85A (139 $\mu \mathrm{M})$ and the mutant Cbl-b peptide $(1084 \mu \mathrm{M})$. Insets show the raw data of each titration. The optimal fits for these two titrations give a $\mathrm{K}_{\mathrm{d}}$ of $1.7 \mu \mathrm{M}$ and an $\mathrm{N}$ of 0.61 for the wildtype peptide and a $\mathrm{K}_{\mathrm{d}}$ of $3.6 \mu \mathrm{M}$ and an $\mathrm{N}$ of 1.27 for the mutant R904A peptide. To better illustrate the different stoichiometries between these two titrations the x-axes are shown on the same scale. The dissociation constant determined for the CIN85A/Cbl-b complex is only an apparent $\mathrm{K}_{\mathrm{d}}$ as it includes binding as well as trimerization.

Figure 4. $\mathrm{c}-\mathrm{Cbl}$ and $\mathrm{Cbl-b}$ mediate ternary complex formation with CIN85 in vivo. (a) HEK293T cells transfected with FLAG-CIN85-3SH3, full length CIN85 and c-Cbl-849 or c-Cbl-827 were lysed and immunoprecipitated by anti-FLAG affinity agarose beads. Total cell lysates (TCL) and immunoprecipitates (IP) were subjected to immunoblotting (WB) with corresponding antibodies. (b) Cbl-b-927 or Cbl-b-927-R904A were transfected along with FLAG-CIN85-3SH3 and full length CIN85 in HEK293T cells and cell lysates were analyzed as described in Fig. 4a. (c) Transfection and immunoprecipitations were done as in Fig. $4 \mathbf{a}$ and b. Immunoblots from at least three independent experiments were then quantified to assess Cbl/CIN85 trimerization. (d) HEK293T cells were transfected with the indicated constructs and subjected to similar treatment as above. Co-immunoprecipitated $\mathrm{c}-\mathrm{Cbl}$ or $\mathrm{Cbl}-\mathrm{b}$ from at least three independent experiments was quantified by ImageJ software. (e) Mutation of Arg822 and Arg829 impairs the ability of c-Cbl-849 to promote EGFR degradation. Total EGFR levels were determined by flow cytometry in HEK293T cells expressing EGFR- 
GFP and the indicated c-Cbl mutants. (f) Mutation of Arg904 and Arg911 impairs the ability of Cbl-b-927 to promote EGFR degradation.

Figure 5. Comparison of the ternary $\beta$-PIX/Cbl-b complex with a SuperSH3 domain. Stereo representation of an overlap of the $\beta$-PIX ${ }_{B} / C b l-b$ complex (in green) with the p47-p22 phox structure (in orange). The peptides are shown in a ball and stick representation. 
Table 1 Peptide binding of the SH3 domains of PIX and CIN85A

\begin{tabular}{cccc}
\hline & $\boldsymbol{N}$ & $\boldsymbol{K}_{\boldsymbol{d}}(\boldsymbol{\mu M})$ & $\Delta \boldsymbol{H}(\mathbf{k c a l} / \mathbf{m o l})$ \\
\hline $\boldsymbol{\beta}$-PIX SH3 + Cbl-b & $0.57 \pm 0.1$ & $14.0 \pm 3.5$ & $-8.35 \pm 1.2$ \\
CIN85A + Cbl-b & $0.57 \pm 0.06$ & $2.5 \pm 1.0$ & $-14.25 \pm 0.5$ \\
$\boldsymbol{\beta}$-PIX SH3 + Pak & $0.95 \pm 0.07$ & $3.2 \pm 0.3$ & $-16.75 \pm 0.3$ \\
CIN85A + Pak & $0.92 \pm 0.004$ & $68.0 \pm 2.0$ & $-9.8 \pm 0.1$ \\
$\boldsymbol{\beta}$-PIX SH3 + mutant Cbl-b & $0.75 \pm 0.27$ & $140.0 \pm 30.0$ & $-13.3 \pm 5.7$ \\
CIN85A + mutant Cbl-b & $1.24 \pm 0.03$ & $4.4 \pm 1.1$ & $-7.9 \pm 1.6$ \\
\hline
\end{tabular}

All titrations apart from CIN85A/Pak and $\beta$-PIX SH3/mutant Cbl-b were carried out between 2-4 times and the estimated error is the standard deviation between measurements. The latter two titrations were carried out only once due to the high protein and peptide concentrations required for these weak interactions. The error given for these titrations is the error of the respective fit. 
Table 2 Data collection and refinement statistics

\begin{tabular}{lll}
\hline & $\beta-\mathrm{PIX} / \mathrm{Cbl}-\mathrm{b}$ & $\mathrm{CIN} 85-\mathrm{SH} 3 \mathrm{~A} / \mathrm{Cbl}-\mathrm{b}$ \\
\hline $\begin{array}{l}\text { Data collection } \\
\text { Space group }\end{array}$ & & \\
Cell dimensions & $\mathrm{P} 3_{2} 21$ & $\mathrm{P} 6_{5}$ \\
$\quad a, b, c(\AA)$ & $69.34,69.34,58.11$ & $51.16,51.16,97.47$ \\
Resolution $(\AA)$ & $26.2-1.85(1.93-1.85)$ & $20.0-2.0(2.07-2.00)$ \\
$R_{\text {sym }}$ & $0.086(0.664)$ & $0.062(0.430)$ \\
$I / \sigma I$ & $31.8(2.94)$ & $29.0(2.87)$ \\
Completeness $(\%)$ & $99.5(98.1)$ & $99.7(98.9)$ \\
Redundancy & 9.7 & 5.0 \\
& & \\
Refinement & & $20.0-2.0$ \\
Resolution $(\AA)$ & $26.2-1.85$ & 9,742 \\
No. reflections & 13,340 & $22.1 / 27.1$ \\
$R_{\text {work }} / R_{\text {free }}$ & $22.7 / 26.9$ & \\
No. atoms & & 931 \\
$\quad$ Protein & 982 & $94 / 2$ \\
$\quad$ Ligand/ion & 70 & 82 \\
$\quad$ Water & 164 & 34.99 \\
$B$-factors & & 25.88 \\
$\quad$ Protein & 29.23 & 31.92 \\
$\quad$ Ligand/ion & 39.91 & 0.007 \\
$\quad$ Water & 42.41 & 1.334 \\
R.m.s. deviations & & \\
$\quad$ Bond lengths $(\AA)$ & 0.005 & \\
$\quad$ Bond angles $\left({ }^{\circ}\right)$ & 1.044 & \\
\hline
\end{tabular}

One crystal was used per data set. Highest resolution shell is shown in parenthesis. 


\section{REFERENCES}

1. Schlessinger, J. Ligand-induced, receptor-mediated dimerization and activation of EGF receptor. Cell 110, 669-72 (2002).

2. Dikic, I. \& Giordano, S. Negative receptor signalling. Curr Opin Cell Biol 15, 128-35 (2003).

3. Bonifacino, J.S. \& Traub, L.M. Signals for sorting of transmembrane proteins to endosomes and lysosomes. Annu Rev Biochem 72, 395-447 (2003).

4. Szymkiewicz, I., Shupliakov, O. \& Dikic, I. Cargo- and compartment-selective endocytic scaffold proteins. Biochem J 383, 1-11 (2004).

5. Sigismund, S. et al. Clathrin-independent endocytosis of ubiquitinated cargos. Proc Natl Acad Sci U S A 102, 2760-5 (2005).

6. Le Roy, C. \& Wrana, J.L. Clathrin- and non-clathrin-mediated endocytic regulation of cell signalling. Nat Rev Mol Cell Biol 6, 112-26 (2005).

7. Haglund, K., Di Fiore, P.P. \& Dikic, I. Distinct monoubiquitin signals in receptor endocytosis. Trends Biochem Sci 28, 598-603 (2003).

8. Haglund, K. et al. Multiple monoubiquitination of RTKs is sufficient for their endocytosis and degradation. Nat Cell Biol 5, 461-6 (2003).

9. Mosesson, Y. et al. Endocytosis of receptor tyrosine kinases is driven by monoubiquitylation, not polyubiquitylation. J Biol Chem 278, 21323-6 (2003).

10. Soubeyran, P., Kowanetz, K., Szymkiewicz, I., Langdon, W.Y. \& Dikic, I. CblCIN85-endophilin complex mediates ligand-induced downregulation of EGF receptors. Nature 416, 183-7 (2002).

11. Petrelli, A. et al. The endophilin-CIN85-Cbl complex mediates ligand-dependent downregulation of c-Met. Nature 416, 187-90 (2002). 
12. Szymkiewicz, I. et al. CIN85 participates in Cbl-b-mediated down-regulation of receptor tyrosine kinases. J Biol Chem 277, 39666-72 (2002).

13. Kowanetz, K. et al. Identification of a novel proline-arginine motif involved in CIN85-dependent clustering of $\mathrm{Cbl}$ and down-regulation of epidermal growth factor receptors. J Biol Chem 278, 39735-46 (2003).

14. Kurakin, A.V., Wu, S. \& Bredesen, D.E. Atypical recognition consensus of CIN85/SETA/Ruk SH3 domains revealed by target-assisted iterative screening. J Biol Chem 278, 34102-9 (2003).

15. Manser, E. et al. PAK kinases are directly coupled to the PIX family of nucleotide exchange factors. Mol Cell 1, 183-92 (1998).

16. Bagrodia, S., Taylor, S.J., Jordon, K.A., Van Aelst, L. \& Cerione, R.A. A novel regulator of p21-activated kinases. J Biol Chem 273, 23633-6 (1998).

17. Etienne-Manneville, S. \& Hall, A. Rho GTPases in cell biology. Nature 420, 629-35 (2002).

18. Flanders, J.A. et al. The Cbl proteins are binding partners for the Cool/Pix family of p21-activated kinase-binding proteins. FEBS Lett 550, 119-23 (2003).

19. Wu, W.J., Tu, S. \& Cerione, R.A. Activated Cdc42 sequesters c-Cbl and prevents EGF receptor degradation. Cell 114, 715-25 (2003).

20. Mayer, B.J. SH3 domains: complexity in moderation. J Cell Sci 114, 1253-63 (2001).

21. Dalgarno, D.C., Botfield, M.C. \& Rickles, R.J. SH3 domains and drug design: ligands, structure, and biological function. Biopolymers 43, 383-400 (1997).

22. Kuriyan, J. \& Cowburn, D. Modular peptide recognition domains in eukaryotic signaling. Annu Rev Biophys Biomol Struct 26, 259-88 (1997). 
23. Musacchio, A. How SH3 domains recognize proline. Adv Protein Chem 61, 21168 (2002).

24. Zarrinpar, A., Bhattacharyya, R.P. \& Lim, W.A. The structure and function of proline recognition domains. Sci STKE 2003, RE8 (2003).

25. Feng, S., Chen, J.K., Yu, H., Simon, J.A. \& Schreiber, S.L. Two binding orientations for peptides to the Src SH3 domain: development of a general model for SH3-ligand interactions. Science 266, 1241-7 (1994).

26. Lim, W.A., Richards, F.M. \& Fox, R.O. Structural determinants of peptidebinding orientation and of sequence specificity in SH3 domains. Nature 372, 375-9 (1994).

27. Groemping, Y., Lapouge, K., Smerdon, S.J. \& Rittinger, K. Molecular basis of phosphorylation-induced activation of the NADPH oxidase. Cell 113, 343-55 (2003).

28. Bartkiewicz, M., Houghton, A. \& Baron, R. Leucine zipper-mediated homodimerization of the adaptor protein c-Cbl. A role in c-Cbl's tyrosine phosphorylation and its association with epidermal growth factor receptor. $J$ Biol Chem 274, 30887-95 (1999).

29. Dikic, I. Mechanisms controlling EGF receptor endocytosis and degradation. Biochem Soc Trans 31, 1178-81 (2003).

30. Kowanetz, K. et al. CIN85 associates with multiple effectors controlling intracellular trafficking of epidermal growth factor receptors. Mol Biol Cell 15, 3155-66 (2004).

31. Watanabe, S. et al. Characterization of the CIN85 adaptor protein and identification of components involved in CIN85 complexes. Biochem Biophys Res Commun 278, 167-74 (2000). 
32. Wiseman, T., Williston, S., Brandts, J.F. \& Lin, L.N. Rapid measurement of binding constants and heats of binding using a new titration calorimeter. Anal Biochem. 179, 131-137 (1989).

33. Otwinowski, Z., and Minor, W. Processing of X-ray diffraction data collected in oscillation mode. in Methods Enzymol., Vol. 276 307-326 (1997).

34. Vagin, A. \& Teplyakov, A. An approach to multi-copy search in molecular replacement. Acta Crystallogr D Biol Crystallogr 56 Pt 12, 1622-4 (2000).

35. Murshudov, G.N., Vagin, A.A. \& Dodson, E.J. Refinement of macromolecular structures by the maximum-likelihood method. Acta Crystallogr D Biol Crystallogr 53, 240-55 (1997).

36. Perrakis, A., Harkiolaki, M., Wilson, K.S. \& Lamzin, V.S. ARP/wARP and molecular replacement. Acta Crystallogr D Biol Crystallogr 57, 1445-50 (2001).

37. Turk, D. Technische Universitaet (1992). Further Development of a Program for Molecular Graphics and Electron Density Manipulation and Its Use in Different Protein Structure Determinations. PhD Thesis, Technische Universitaet (1992).

38. Laskowski, R.A., Moss, D.S. \& Thornton, J.M. Main-chain bond lengths and bond angles in protein structures. J Mol Biol 231, 1049-67 (1993).

39. Navaza, J. AMoRe: an automated package for molecular replacement. Acta Crystallogr. A 50, 157-163 (1994).

40. Brunger, A.T. et al. Crystallography \& NMR system: A new software suite for macromolecular structure determination. Acta Crystallogr D Biol Crystallogr 54 ( Pt 5), 905-21 (1998).

41. Emsley, P. \& Cowtan, K. Coot: model-building tools for molecular graphics. Acta Crystallogr D Biol Crystallogr 60, 2126-32 (2004). 
42. McRee, D.E. XtalView/Xfit--A versatile program for manipulating atomic coordinates and electron density. J Struct Biol 125, 156-65 (1999).

43. Abramoff, M.D., Magelhaes, P.J. \& Ram, S.J. Image processing with ImageJ. Biophotonics Internatinal 11, 36-42 (2004). 
a

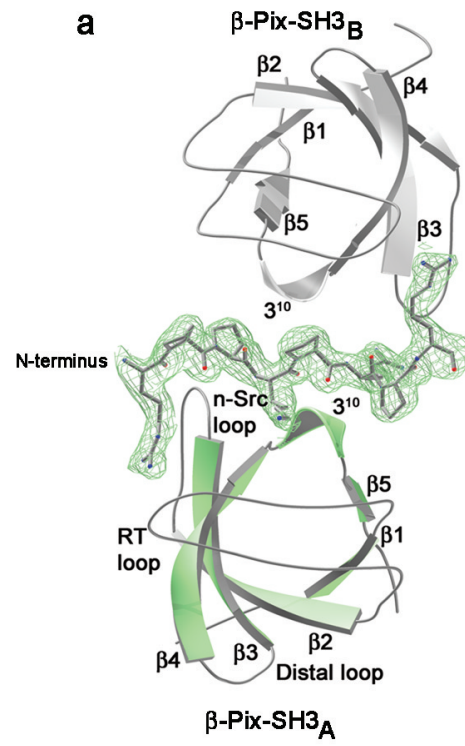

b $\quad$ CIN85-SH3 3

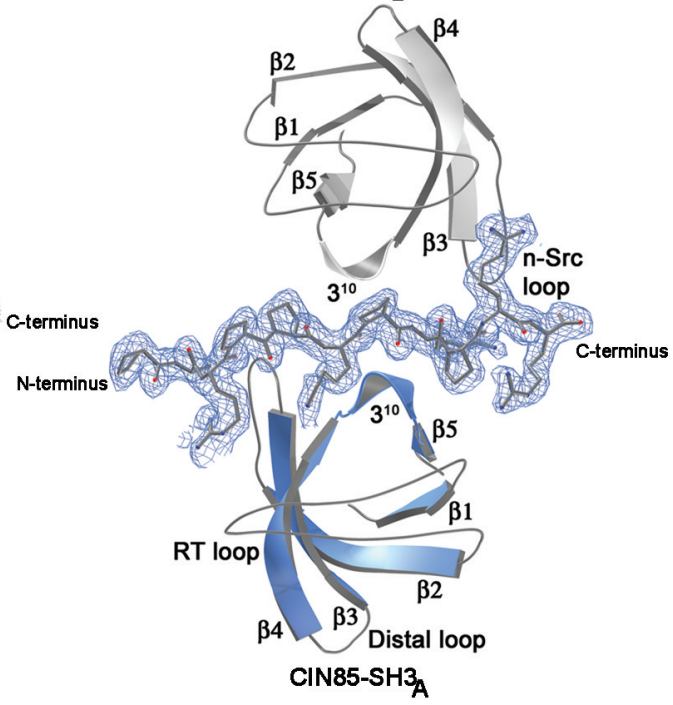

C
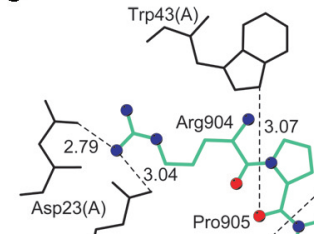

Pro56(A)痣 Glu24(A)

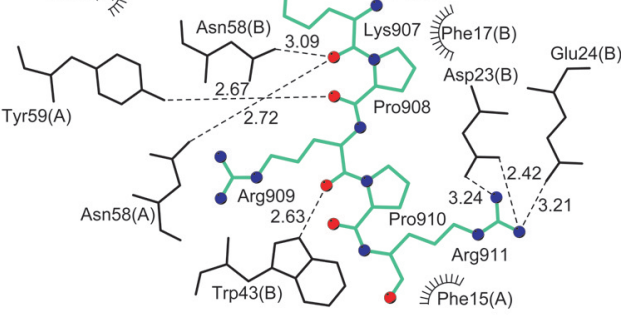

d

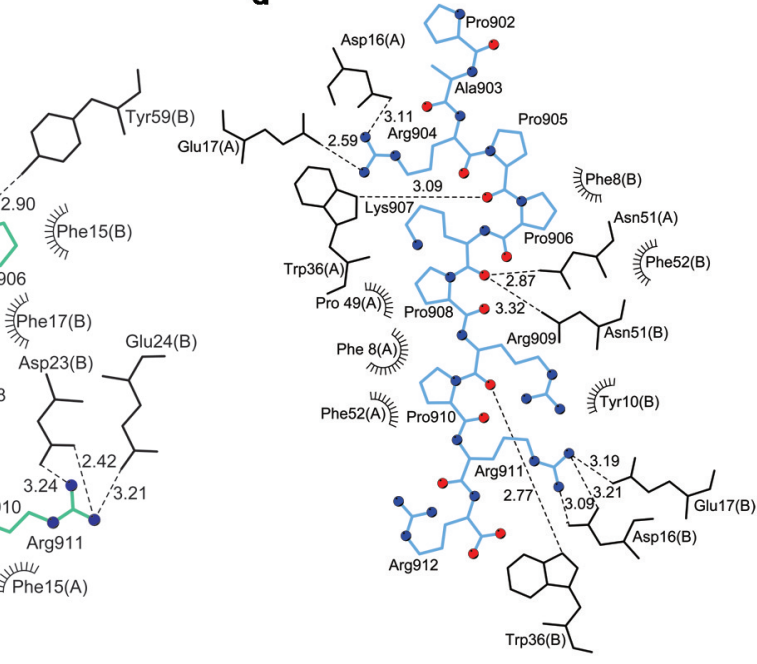



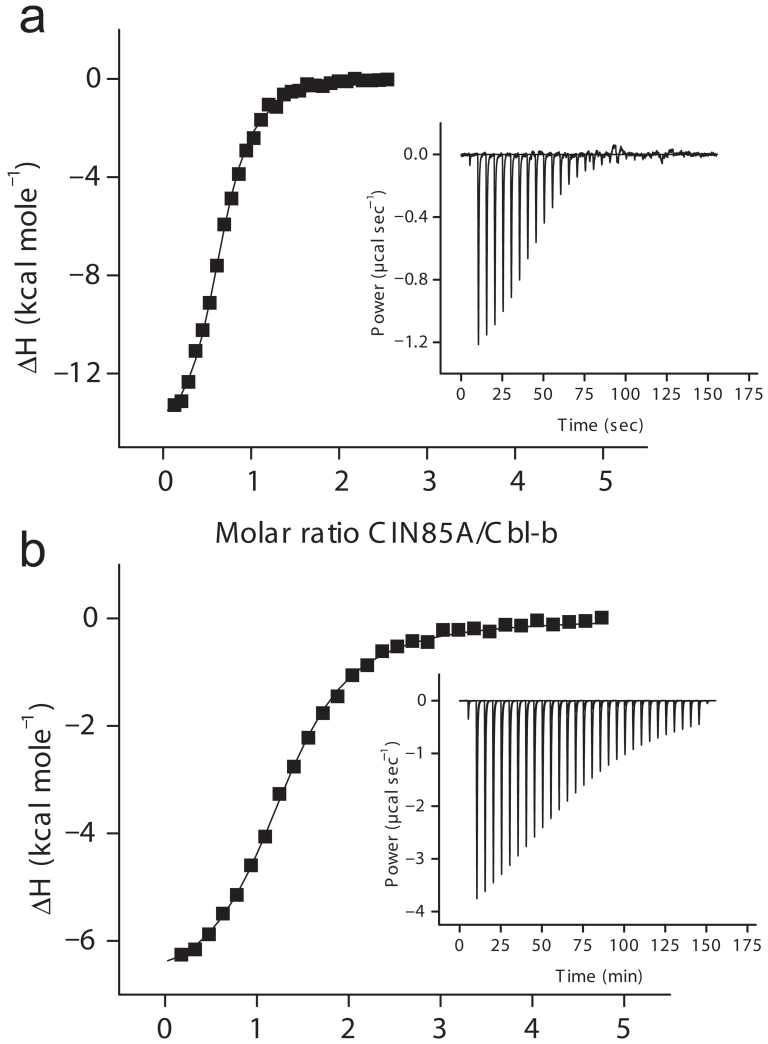

Molar ratio $\mathrm{CIN} 85 \mathrm{~A} / \mathrm{C}$ bl-b mutant 
C

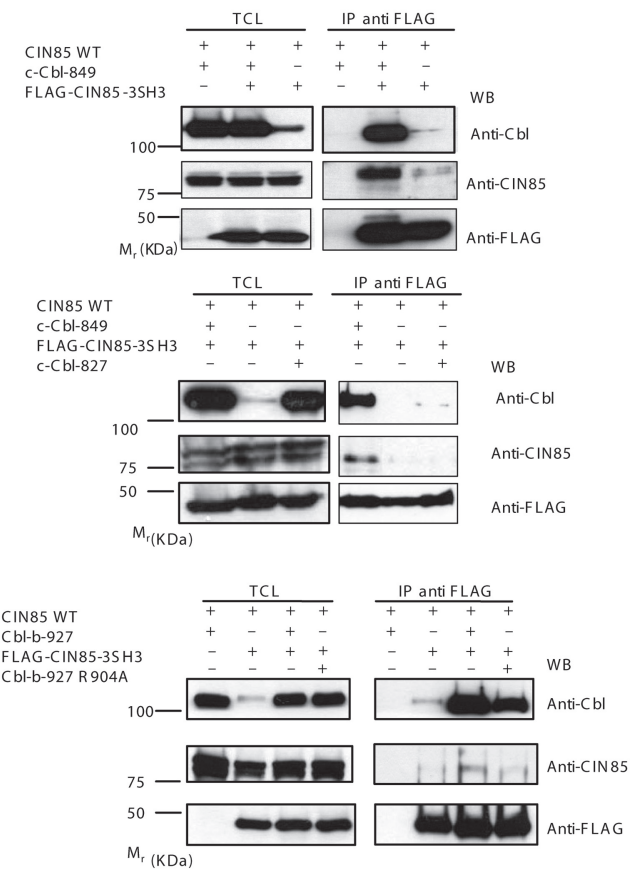

d
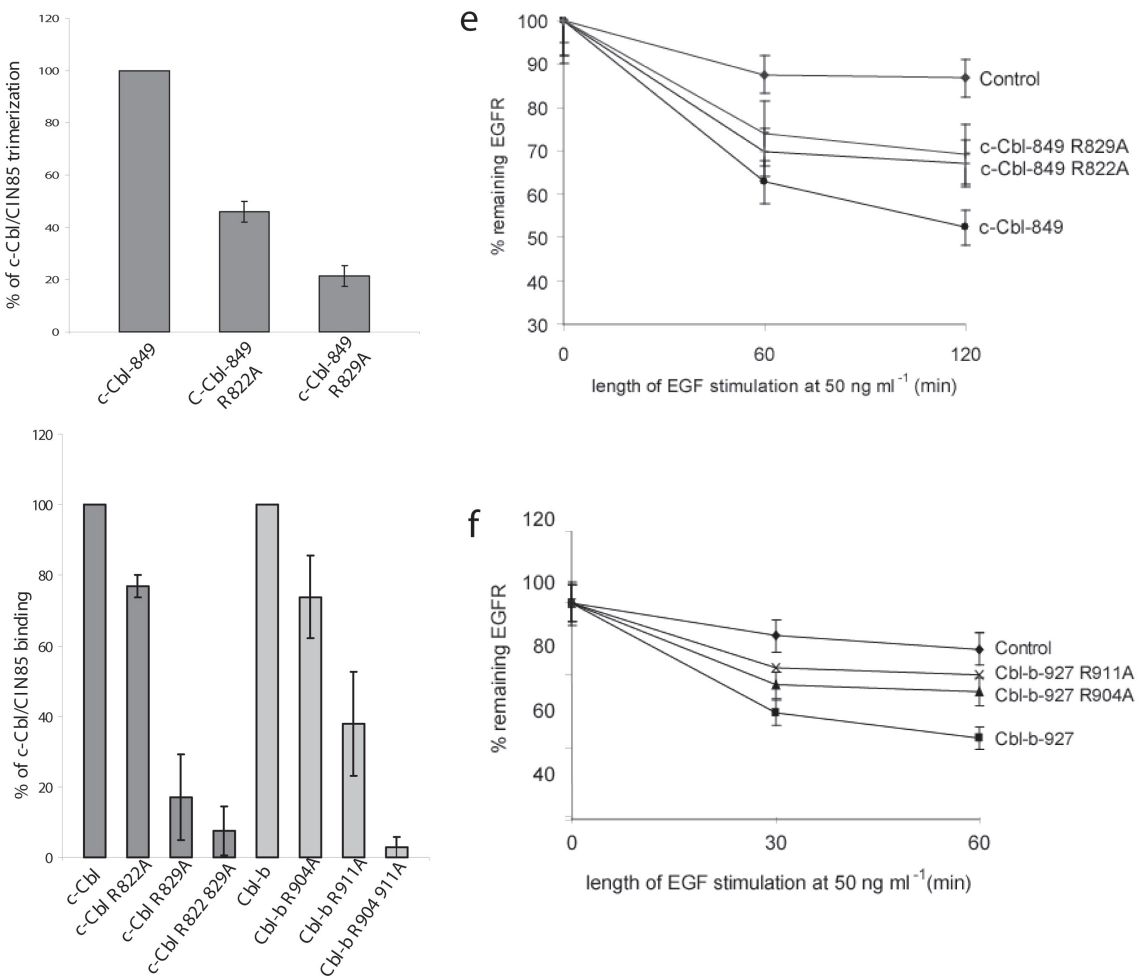

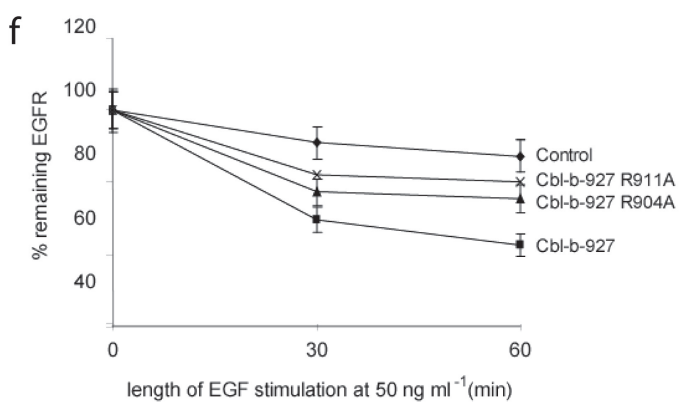


